\title{
Finding an Objective Cost for Sliceable Flexgrid Transponders
}

\author{
L. Velasco ${ }^{1 *}$, O. González de Dios ${ }^{2}$, V. López ${ }^{2}$, J. Fernández-Palacios ${ }^{2}$, and G. Junyent ${ }^{1}$ \\ ${ }^{1}$ Optical Communications Group (GCO). Universitat Politècnica de Catalunya (UPC), Barcelona, Spain \\ ${ }^{2}$ Telefónica, Investigación y Desarrollo, Madrid, Spain \\ *e-mail:lvelasco@ac.upc.edu
}

\begin{abstract}
We analyze the objective cost of SBVTs as a function of the traffic conveyed by IP/MPLS-over-flexgrid networks. Optimal results show that cost increment with respect to BVTs is related to the aggregation level.

(C) 2014 Optical Society of America

OCIS codes: (060.0060) Fiber optics and optical communications; (060.4250) Networks
\end{abstract}

\section{Introduction}

National IP/MPLS networks receive IP traffic from access networks and perform aggregation and routing. To minimize the number of ports a router hierarchy consisting of edge routers performing aggregation and transit routers providing routing flexibility is typically created. In addition, a reduced number of interconnection routers support inter-operator connections. For efficiency and reach reasons, IP/MPLS networks are usually designed on top of optical networks [1]. The maturity of the flexgrid technology [2] providing fine spectral granularity makes bandwidth-variable optical cross-connects (BV-OXCs) based on that technology the candidates to be deployed in operators networks in the near future (Fig. 1).

To take advantage from the mix of optical connection (lightpath) bitrates that can be created on flexgrid networks, bandwidth-variable optical transponders (BVT) can be used. The main advantages of BVTs with respect fixed transponders (FTP) are: $i$ ) the used spectrum width is a function of the actual lightpath's bitrate and $\mathrm{i}$ ) procurement and inventory is simplified, thus reducing operational expenditures (OPEX). However, when the associated lightpath's bitrate is low, BVT's capacity is wasted, thus increasing capital expenditures (CAPEX). For instance, imagine a $400 \mathrm{~Gb} / \mathrm{s}$ BVT currently used for a 100Gb/s lightpath. Notwithstanding, to ensure that the network can support the traffic forecast for the years to come, deploying transponders with lower capacity might also involve increasing CAPEX to replace those transponders in the near future. The answer to that dilemma is sharing each BVT among several lightpaths, as proposed in [3]. A sliceable bandwidth-variable optical transponder (SBVT) is thus a BVT that can be shared by multiple $(f)$ lightpaths.

We assume that IP/MPLS routers are connected to wavelength selective switches (WSS) in the local BV$\mathrm{OXC}$, similarly to the architecture in [4]. Virtual topologies are created by connecting IP/MPLS routers among them through lightpaths established in the flexgrid optical layer. IP/MPLS routers are dimensioned considering two main parameters: forwarding capacity and number of slots available in the chassis, which limits the number of cards that can be installed. Since the bitrate of the MPLS flows resulting from aggregating IP client flows at edge routers is usually low, those routers are connected to few transit routers and BVTs or FTPs are usually preferred. However, the connectivity of transit routers among other transit and interconnection routers is usually high, creating an almost full-mesh virtual topology among them (Fig. 2). Therefore, SBVTs could provide advantages by reducing the number of cards to be installed on transit and interconnection routers, thus saving CAPEX since cheaper routers could need to be installed.

In this paper, we focus on dimensioning transit and interconnection routers since installing SBVTs instead of FTP or BVTs could reduce the cost of those routers. To avoid compatibility problems, we assume that BVTs

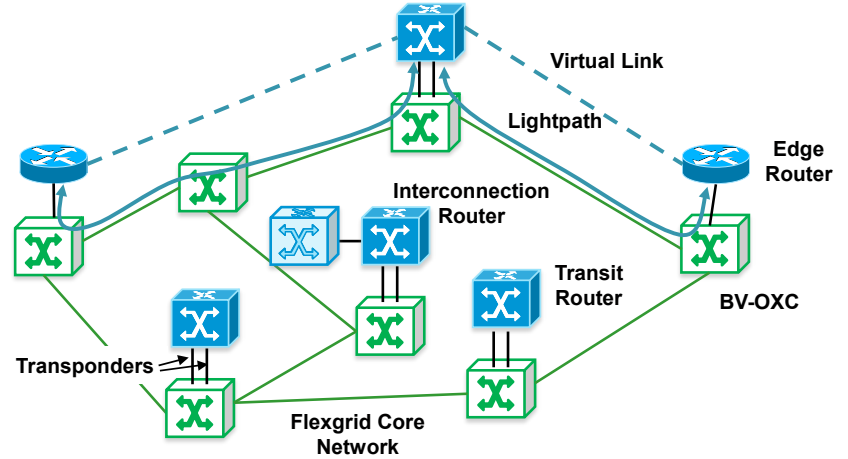

Fig. 1 Example of a multilayer IP/MPLS-over-Flexgrid network.

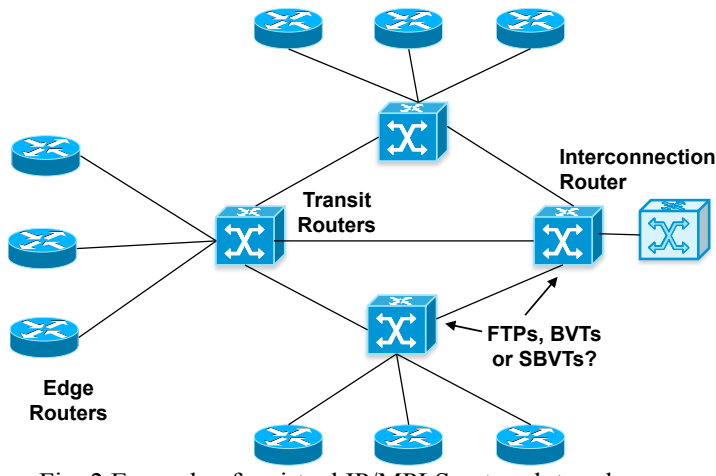

Fig. 2 Example of a virtual IP/MPLS network topology.

The research leading to these results has received funding from the European Community's Seventh Framework Programme FP7/2007-2013 under grant agreement $\mathrm{n}^{\circ} 317999$ IDEALIST project and by the Spanish MINECO project ELASTIC (TEC2011-27310). 
can be installed in edge routers when required. We study the implications of using SBVTs and their impact in network CAPEX for different network scenarios and find the objective price of SBVTs as a function of $f$.

\section{IP/MPLS-over-Flexgrid Dimensioning (IPoF) problem}

The IPoF problem can be formally stated as:

Given: $i$ ) a network topology represented by a graph $G(N, E)$, being $N$ the set of locations where BV-OXCs are installed and $E$ the set of fiber links connecting two locations in $N$; ii) the subsets $N_{E} \subseteq N, N_{T} \subseteq N$, and $N_{I} \subseteq N$, where edge, transit and interconnection IP/MPLS routers are installed, respectively; iii) the characteristics of the optical spectrum (i.e., width, frequency slot width) and the used modulation format.; $i v$ ) a traffic matrix $D$ with the amount of bitrate exchanged between each pair of locations in $N ; v$ ) the cost of every component, including IP/MPLS routers, router cards, optical components (WSSs and optical amplifiers, OAs) to build BV-OXCs, and TPs specifying its type (FTP, BVT or SBVT), capacity, and number of flows (BVTs $f=1$ and SBVTs $f>1$ ).

Output: i) The network dimensioning, including: a) WSSs, OAs and nodal degree for each BV-OXC; b) the amount and type of used TPs in each router; c) the type of IP/MPLS router in each location. ii) The route on the virtual topology or the router and spectrum allocation on the flexgrid network for each demand in $D$.

Objective: Minimize the total CAPEX (IP/MPLS routers, TPs, and BV-OXCs) to transport the given traffic $D$.

The costs of IP/MPLS routers, WSSs, OAs, and both, FTPs and BVTs is known [5]. To find the objective cost of SBVTs, we solved the IPoF problem for several values of $f$ assuming that the cost of SBVTs is equal to that of the BVTs with the same capacity and compared solutions' CAPEX using equation (1). Note that, by definition, $\Delta \cos t \geq 0$; when $\Delta$ cost $=0$ the cost of a SBVT and a BVT are the same.

$$
\Delta \operatorname{cost}=\frac{\operatorname{CAPEX}(f=1)-\operatorname{CAPEX}(f>1)}{\operatorname{CostBVT} \cdot \operatorname{Num} T P s}, \quad \operatorname{CostBVT}=\kappa \quad \forall f
$$

\section{Illustrative numerical results}

In this section, we present the obtained results from solving problem instances for an IP/MPLS network with 180 locations and 373 fiber links, based on the Telefónica's national network. The traffic matrix was generated by randomly selecting two types of bidirectional IP flows: a) 16,110 internal network flows between every IP/MPLS edge router pairs in the continuous range $[1,400] \mathrm{Mb} / \mathrm{s}$, and b) 180 interconnection flows from every IP/MPLS edge router in the continuous range $[1,10] \mathrm{Gb} / \mathrm{s}$; so as to obtain a traffic matrix with $4.2 \mathrm{~Tb} / \mathrm{s}$. To emulate the traffic increment foreseen for the years to come, we applied a multiplier to each flow individually to obtain 5.0, 6.7, and $8.4 \mathrm{~Tb} / \mathrm{s}$ matrices. Five different multilayer topologies where created by increasing the number of transit routers $\left(\left|N_{T}\right|\right)$ from 10 to 18 , while keeping constant the location of edge routers. We assume 6.25 GHz spectrum granularity and $4 \mathrm{THz}$ of optical spectrum for the flexgrid network. As for the TPs, this study concentrates on $400 \mathrm{~Gb} / \mathrm{s}$ capacity (S)BVTs and considers values for $f$ in the range [1-10]. For comparison purposes, also the solution where only FTPs was used, labeled as Mixed Line Rate (MLR). We assume that three different cards and TPs can be used: $14 \times 10 \mathrm{~Gb} / \mathrm{s}, 3 \times 40 \mathrm{~Gb} / \mathrm{s}, 4 \times 100 \mathrm{~Gb} / \mathrm{s}$ FTPs, and 1x400Gb/s (S)BVTs.

Fig. 3a plots the amount of TPs vs. the number of transit routers for all four traffic matrices. The number of TPs is clearly reduced when SBVTs are used with respect to using BVTs and FTPs, since several lightpaths share the same TP. The optimal value of $f$, however, depends on the size of the traffic matrix. When the traffic matrix is low, lightpaths connecting transit routers are about $60 \mathrm{~Gb} / \mathrm{s}$ on average, which facilitates TP sharing
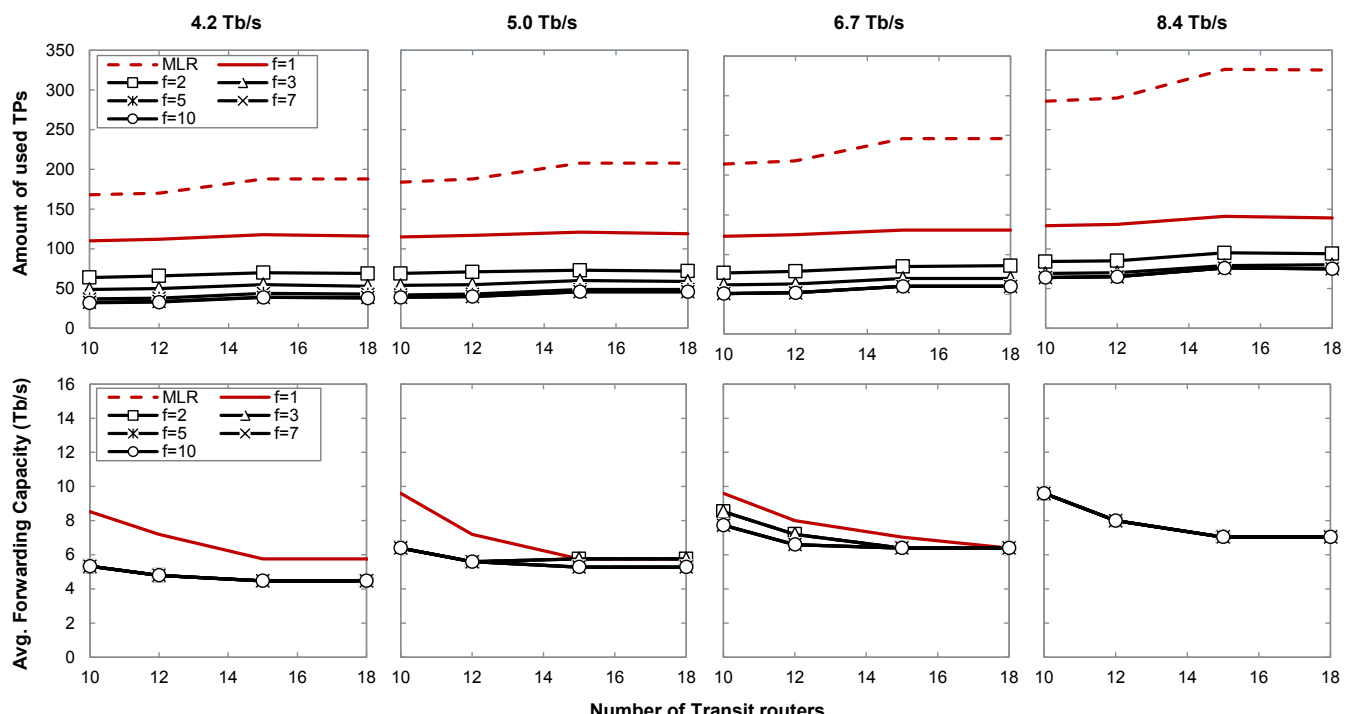

Fig. 3. Amount of TPs (up) and capacity of the transit routers (bottom) against the number of transit routers 

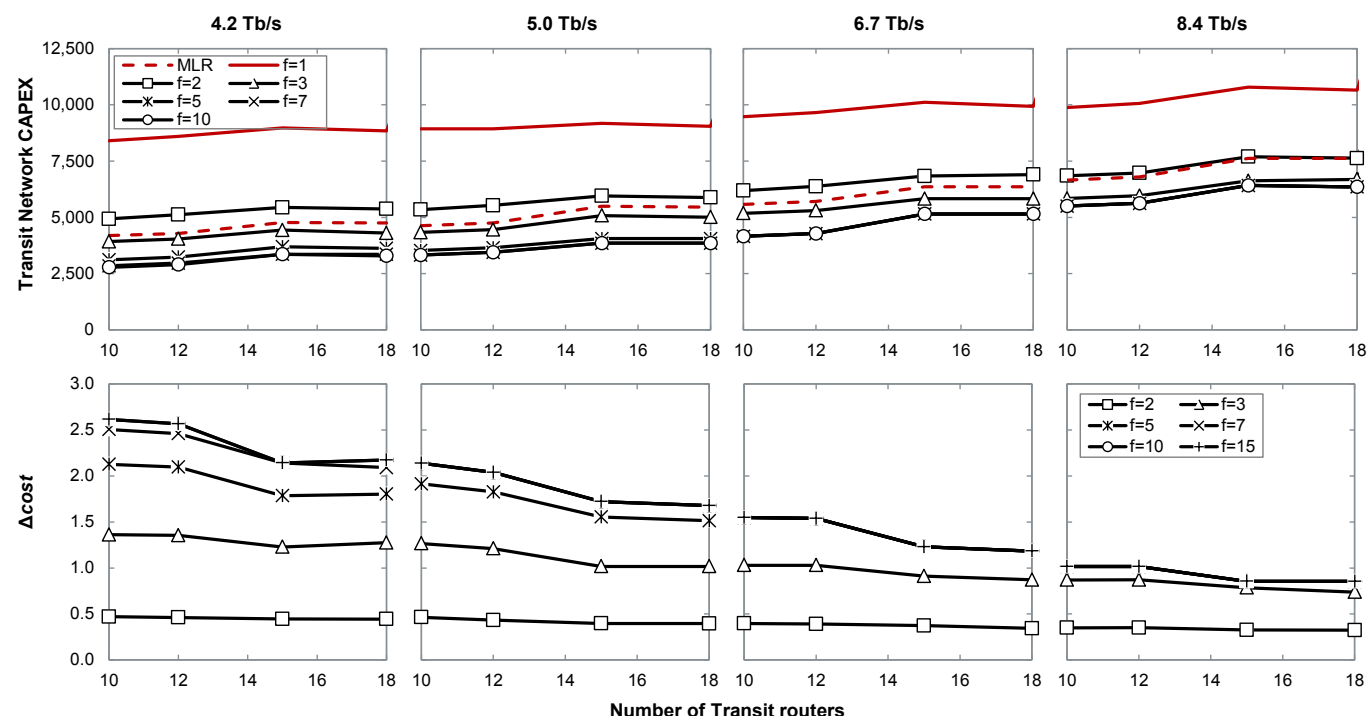

Fig. 4. Total IP/MPLS transit cost (up) and $\Delta$ cost (bottom) against the number of transit routers

and makes the optimal value of $f=7$. However, as soon as the traffic increases, the optimal $f$ decreases as a consequence of the lightpaths' capacity increases, becoming $f=5$ for the largest traffic matrix. Regarding the solution for MLR, the number of TPs used is the largest for all four matrices. Noticeable is the increment in the amount of used TPs with the load for the MLR solutions, in contrast to the more moderated increments obtained when BVTs and SBVTs were used.

Fig. $3 \mathrm{~b}$ plots the installed transit router forwarding capacity. As shown, the installed capacity is remarkably lower when SBVTs are used, as a result of removing over-dimensioning coming from the amount of interfaces needed in each router. As soon as the number of transit routers increases, the total capacity gradually decreases as a result of more lightpaths with lower capacity are established in the network. The benefits of installing SBVTs are virtually eliminated when the traffic volume increases, since routers capacity can be kept constrained by the switching requirements instead of by the size of the chassis and the number of required cards. As for the MLR solutions, the installed capacity was similar to that for the SBVTs, since several FTPs can be installed in one single router card, e.g., $4 \times 100 \mathrm{~Gb} / \mathrm{s}$. It is worth highlighting that the installed capacity would be considerably higher in case of using $2 \mathrm{x}$ or $1 \mathrm{x} 100 \mathrm{~Gb} / \mathrm{s}$ cards.

The costs saved from reducing the installed number of SBVTs and from decreasing the forwarding capacity of transit routers gives room to increment the cost of SBVTs with respect to BVTs. Fig. 4a presents total transit network CAPEX. Interestingly, even using $f=2$ SBVTs CAPEX is dramatically reduced. That reduction is even larger when $f=10$ SBVTs are installed in the case of the lighter traffic matrix or $f=5$ for the heaviest traffic. When using FTPs, CAPEX is between to that of the SBVTs with $f=2$ and $f=3$; this is clearly worse that when SBVTs with $f=4$ are used, which is the case $4 \times 100 \mathrm{~Gb} / \mathrm{s}$ FTP cards should be compared against.

Finally, Fig. 4b plots $\Delta$ cost (computed as eq. 1) against the number of transit routers. As shown, the cost of SBVTs with $f=2$ could be increased almost $50 \%$ over BVTs cost for the transit CAPEX to be equal. $\Delta c o s t$ decrease with the traffic volume and tend to converge and collapse resulting in values just under $1(100 \%$ cost increment) for SBVTs with $f>5$ under the heaviest traffic

\section{Conclusions}

The objective cost of SBVTs has been studied in comparison to that of BVTs as a function of the traffic matrix and the aggregation level obtained by using different sizes of IP/MPLS transit network. Costs saving coming from a reduction in the cost of IP/MPLS routers can be obtained increasing the value of $f$, as a result of reducing the amount of cards needed to be installed in routers chassis. That cost reduction becomes the margin in the increment of SBVT cost.

From the results, we can conclude that there is not a single optimal value of $f$, being that a function of the capacity of the lightpaths. Additionally, high $f$ values do not provide additional cost savings when the aggregation level is high; on the contrary, they are highly beneficial in scenario with many low capacity lightpaths.

\section{References}

1. M. Ruiz et al., "Survivable IP/MPLS-Over-WSON Multilayer Network Optimization,” IEEE/OSA JOCN, vol. 3, 2011.

2. M. Jinno et al., "Spectrum-efficient and scalable elastic optical path network," IEEE Commun Mag., vol. 47, 2009.

3. M. Jinno et al., "Multiflow Optical Transponder for Efficient Multilayer Optical Networking," IEEE Comm. Mag., vol. 50, 2012.

4. T. Tanaka et al., "Impact of Multi-flow Transponder on Equipment Requirements in IP over Elastic Optical Networks," in ECOC 2013.

5. F. Rambach et al., "A Multilayer Cost Model for Metro/Core Networks," IEEE/OSA JOCN, vol. 5, 2013. 